Revue des patrimoines

43 | 2021

Des écoles d'art académiques aux écoles d'art : des collections et des lieux, un patrimoine à valoriser

\title{
La separazione delle pinacoteche dalle accademie di Belle Arti
}

Patrimonio museale e istruzione artistica in Italia intorno al 1882

\section{Martina Lerda}

\section{OpenEdition \\ Journals}

Edizione digitale

URL: http://journals.openedition.org/insitu/29198

DOI: 10.4000/insitu.29198

ISSN: $1630-7305$

\section{Editore}

Ministère de la Culture

Notizia bibliografica digitale

Martina Lerda, «La separazione delle pinacoteche dalle accademie di Belle Arti », In Situ [En ligne], 43 | 2021, mis en ligne le 22 janvier 2021, consulté le 22 janvier 2021. URL : http://

journals.openedition.org/insitu/29198; DOI : https://doi.org/10.4000/insitu.29198

Questo documento è stato generato automaticamente il 22 janvier 2021.

\section{cc)}

In Situ Revues des patrimoines est mis à disposition selon les termes de la licence Creative Commons Attribution - Pas d'Utilisation Commerciale - Pas de Modification 4.0 International. 


\title{
La separazione delle pinacoteche dalle accademie di Belle Arti
}

Patrimonio museale e istruzione artistica in Italia intorno al 1882

\author{
Martina Lerda
}

\section{NOTE DELL'AUTORE}

Questo saggio nasce dalla mia tesi di dottorato, "Le pinacoteche statali nell'Italia unita. Politiche museali e affermazione della disciplina storico-artistica (1859-1882)", discussa nel 2015 presso l'Università degli Studi di Udine. Ringrazio sentitamente Donata Levi e Simona Rinaldi per le preziose indicazioni.

pinacoteca, accademie di Belle Arti, Ministero della Pubblica Istruzione, istruzione artistica, connoisseurship

1 All'indomani dell'Unità d'Italia (1861), il panorama dei musei statali, dipendenti dal Ministero della Pubblica Istruzione, si compone delle raccolte appartenute a diversi istituti pubblici ereditati dagli stati preunitari e dalle raccolte formate nei secoli dalle antiche dinastie appena destituite. La configurazione istituzionale assunta da questo ingente patrimonio è risultato delle dinamiche di statalizzazione delle collezioni attuate dai governi provvisori che, tra 1859 e 1860, nelle diverse aree della penisola hanno immediatamente preceduto l'annessione al Regno d'Italia.

2 Al termine di questo processo, solo alcune quadrerie storiche si configurano come pinacoteche autonome, direttamente dipendenti dal Ministero della Pubblica Istruzione: le Regie Gallerie fiorentine (la Galleria degli Uffizi e quella di Palazzo Pitti, che diventa pubblica nel giugno del 1859) ${ }^{1}$ e, a Torino, la Pinacoteca di Casa Savoia, che nel 1860 , cosa per niente scontata, viene statalizzata sull'esempio di quanto disposto in Toscana ${ }^{2}$.

3 A Napoli, la quadreria borbonica, composta da opere provenienti dalla Reggia di Capodimonte e dal Palazzo Francavilla, era invece confluita sin dal 1806, insieme alle altre raccolte dinastiche, nel Palazzo degli Studi, a costituire una raccolta secondaria di 
un museo complessivamente consacrato alla conservazione dei reperti antichi. Quest'ultimo viene dichiarato Museo Nazionale dal governo dittatoriale di Garibaldi nel dicembre del $1860 .^{3}$

4 Diversa ancora la sorte della raccolta estense conservata nel Palazzo Ducale di Modena, che nel 1859 viene posta alle dipendenze della locale Accademia di Belle $\mathrm{Arti}^{4}$. Come già avvenuto per la piccola quadreria del Palazzo Ducale di Lucca ${ }^{5}$, la raccolta modenese si somma alle quadrerie storiche dipendenti da istituti statali di istruzione. Queste sono: la Pinacoteca palermitana, annessa alle raccolte archeologiche dell'Università fino al $1863^{6}$, e le pinacoteche delle accademie di belle arti di Milano, Bologna, Parma e Firenze, a cui dal 1866, con l'annessione del Veneto al Regno d'Italia, si aggiunge quella di Venezia.

5 Alla luce di questa complessa ricostruzione perciò, alla proclamazione dell'Unità d'Italia, la maggior parte delle quadrerie storiche statali risulta annessa alle accademie di belle arti ed è quindi intesa come strumento accessorio all'istruzione artistica.

6 Il decreto del 13 marzo 1882, che separa dal punto di vista amministrativo le raccolte storiche dalle accademie di Belle Arti, interviene in maniera decisiva sull'assetto del patrimonio museale statale: sottrae le pinacoteche alle accademie e le trasforma in istituti autonomi, cioè direttamente dipendenti dal Ministero della Pubblica Istruzione. ${ }^{7}$ Si tratta di una riforma poco studiata, ma tutt'altro che marginale, dato che contribuisce a determinare la fisionomia del moderno sistema museale statale italiano e incide su quello stretto legame tra raccolte pittoriche e formazione artistica che il Regno d'Italia aveva ereditato dagli stati pre-unitari e dai governi di transizione.

7 Questo contributo, che non intende in alcun modo ricostruire le vicende delle singole raccolte accademiche italiane, si concentrerà proprio sul processo di elaborazione e sul significato di questo decisivo provvedimento.

8 La riforma rappresenta indubbiamente una modifica di carattere amministrativo, ma sono innegabili i suoi risvolti anche sul piano culturale. La posta in gioco, infatti, è la funzione assegnata dal livello politico alle pinacoteche storiche, che da questo momento smettono di essere inquadrate come strumenti accessori all'istruzione artistica e allargano la loro destinazione ufficiale.

Il nuovo stato di cose è da considerarsi come il risultato di un processo complesso, in cui agiscono spinte di natura differente: dalle rivendicazioni disciplinari dei primi esponenti della connoisseurship italiana, ai tormentati tentativi di riforma delle accademie di belle arti, sino all'esigenza del neonato stato italiano di dare un'organizzazione amministrativa al suo sistema museale.

10 In primo luogo, la centralità delle pinacoteche nella cultura dei conoscitori ottocenteschi permette una riflessione sui rapporti esistenti tra elaborazione istituzionale e istanze provenienti dagli ambienti più aggiornati della critica storicoartistica. In altre parole: lo studio di politiche relative alle pinacoteche, come quella in oggetto, fornisce un'angolazione inusuale da cui osservare il processo di affermazione in Italia della storia dell'arte come disciplina autonoma. Le dinamiche coinvolte nell'elaborazione del provvedimento verranno quindi indagate a partire dal contributo svolto da alcuni noti conoscitori - in special modo Giovanni Morelli e Giuseppe Mongeri - nella definizione di una più ampia funzione museale delle raccolte pittoriche italiane; il loro contributo è volto in particolar modo all'introduzione di criteri coerenti di ordinamento (spinta che può essere inserita nel quadro delineato da Pomian in 
merito all'ingresso della storia nel museo), ${ }^{8}$ nonché alla professionalizzazione e al riconoscimento delle competenze storico-artistiche necessarie all'amministrazione delle raccolte pittoriche. È in questo contesto che prendono forma le prime proposte per l'autonomia delle pinacoteche.

\section{L'autonomia delle pinacoteche nelle proposte dei conoscitori}

11 L'ambiente dei conoscitori individua nelle pinacoteche uno strumento fondamentale per l'affermazione della nascente disciplina storico-artistica. Di conseguenza, non mancano sin dai primi anni del Regno d'Italia alcune proposte di riorganizzazione delle raccolte, compatibili con i più aggiornati modelli storiografici. Basti ricordare a tal proposito due famosi interventi pubblici: il discorso tenuto da Giovanni Morelli alla Camera nel luglio del $1862^{9}$ e l'articolo di Giovanni Battista Cavalcaselle, pubblicato nel 1863 sulla Rivista dei Comuni italiani ${ }^{10}$. I due interventi denunciano all'unisono la sconfortante condizione delle pinacoteche italiane all'indomani dell'Unità e prospettano soluzioni affini, come la verifica delle attribuzioni, l'aggiornamento dei cataloghi, il completamento e il coerente riordino delle raccolte. Per quanto riguarda quest'ultimo aspetto, Cavalcaselle propende con decisione per il criterio cronologico per scuole pittoriche, più adatto a tradurre visivamente gli sviluppi della storia dell'arte, secondo i metodi d'indagine adottati dalla connoisseurship europea.

12 La rivendicazione disciplinare dei conoscitori si scontra però, inevitabilmente, con la tradizionale autorità esercitata dalle accademie di belle arti nel campo della tutela. Cavalcaselle infatti non risparmia in più occasioni critiche marcate sull'operato di questi istituti, che "mostrano di saper poco dell'arte antica, o fingono di non sapere, o non si occupano ${ }^{11}$." Sarà tuttavia Morelli il primo a mettere concretamente in discussione il ruolo delle accademie di belle arti nella gestione del patrimonio museale. In un progetto di legge dei primi anni Sessanta, poi naufragato, Morelli propone proprio la separazione delle pinacoteche dalle accademie di belle arti, a partire dal presupposto che: "L'arte antica vuole assolutamente essere segregata dall'arte moderna ${ }^{12}$."

13 La distinzione tra funzione conservativa e istruzione artistica implica infatti il fondamentale riconoscimento di nuove competenze professionali, e mira al superamento di quello che un altro conoscitore, Giuseppe Mongeri, definiva come il "pregiudizio troppo comune che mette a fascio l'artista coll'intendente dell'arte antica ${ }^{13}$ ".

Inoltre, la creazione di istituti direttamente dipendenti dal Ministero consentirebbe finalmente un aggiornamento organico delle pinacoteche sul modello delle principali gallerie europee. È quanto emerge da una nuova proposta redatta ancora da Morelli insieme a Mongeri nel febbraio $1870^{14}$. Qui l'autonomia delle pinacoteche costituisce una premessa fondamentale sia per l'adozione di principi uniformi nella gestione degli istituti statali, sia per un radicale ripensamento dell'intero sistema museale nazionale.

15 I due conoscitori immaginano infatti una grande pinacoteca a Firenze, che rappresenti sinteticamente le diverse scuole italiane. Agli istituti diffusi sul territorio nazionale assegnano invece l'onere di illustrare gli sviluppi delle singole scuole pittoriche locali. In quest'ottica Morelli e Mongeri legittimano scambi, accentramenti e ricomposizioni 
di opere smembrate. Il sistema museale ipotizzato risulta così tendenzialmente incurante della stratificazione storica delle collezioni; d'altra parte, però, è certamente funzionale all'affermazione e alla divulgazione della disciplina storico-artistica.

Il Ministro della Pubblica Istruzione Cesare Correnti, culturalmente e politicamente vicino ai due conoscitori, approva le linee generali del progetto, tanto da avanzare alcuni punti della riforma all'interno dei Provvedimenti per il pareggio del Bilancio. L'articolo 8 dell'allegato c, presentato alla Camera il 10 marzo 1870, prevede il trasferimento delle accademie di belle arti a carico dei Comuni. Mentre l'articolo 9 stabilisce che: "Le pinacoteche e le raccolte artistiche ed archeologiche appartenenti allo Stato, ed ancor quelle che sono unite alle accademie e scuole di belle arti, verranno costituite e amministrate separatamente da ogni altro istituto e saranno riordinate in modo che ciascuna di esse rappresenti, più compiutamente che sia possibile, le scuole d'arte e le memorie storiche del paese ove è collocata ${ }^{15}$."

La reazione al progetto da parte del ceto accademico è immediata e dà vita a un esplicito e interessante dibattito sulla funzione delle raccolte di quadri antichi.

\section{Il ruolo delle pinacoteche: due visioni a confronto}

Pochi giorni dopo la presentazione del progetto, il Consiglio accademico milanese trasmette una mozione di protesta al Ministro e alle altre accademie di belle arti del Regno. Il principale obiettivo polemico degli accademici milanesi è la possibile dipendenza comunale dell'istituto. Ma non mancano aspre critiche anche contro l'ipotesi di una Pinacoteca braidense autonoma. Le ragioni a sfavore dell'ipotesi sono due: l'importanza didattica della pinacoteca e la naturale connessione tra raccolta e accademia. Infatti, secondo quanto si legge nel documento, "la separazione della Pinacoteca dalla Accademia sottrarrebbe alla libera azione del Corpo insegnante uno dei più efficaci istrumenti del suo ministero". ${ }^{16}$ Infine, nella lettera che accompagna la mozione, il Presidente dell'Accademia milanese, Carlo Belgiojoso, si oppone a un rigoroso riordinamento in funzione della scuola locale che metterebbe pericolosamente a rischio l'integrità della Pinacoteca di Brera, nata come raccolta di capolavori. Questa, infatti, si troverebbe a dover cedere opere di pregio singolare, come lo Sposalizio della Vergine di Raffaello, in cambio di tasselli di una storia pittorica già ampiamente rappresentata all'esterno dell'istituto.

La visione della Pinacoteca e della sua funzione è evidentemente molto distante da quella dei conoscitori: Belgiojoso non riconosce, infatti, efficacia didattica alla coincidenza tra geografia museale e geografia storico-artistica. Anzi, a suo avviso, l'ordinamento delle collezioni secondo la scuola locale "nuocerebbe alla diffusione della cultura artistica", poiché costringerebbe i giovani artisti a continui spostamenti. Il Presidente sembra piuttosto assegnare utilità didattica ai "proficui apprezzamenti che porge la comparazione di opere di scuole diverse".

20 È questa la concezione museale predominante all'interno del ceto accademico italiano. La mozione di Brera, infatti, è appoggiata dai consigli accademici di Parma, Modena, Bologna e Venezia. Le loro delibere riprendono puntualmente le obiezioni dei milanesi e denunciano la contraddizione tra l'ordinamento previsto dall'articolo 9 e la funzione che si vorrebbe unificante dell'arte nazionale. Così dichiara, per esempio, il Consiglio accademico di Venezia: 
Dare ad esse [le pinacoteche] un colore tutto locale accogliendovi i soli capo-lavori di ogni singola scuola danneggerebbe gli studii togliendo la possibilità dei confronti; offenderebbe il carattere universale che l'arte dee avere; le farebbe perdere quelle nuove qualità, che le impartirono le tolte barriere, la comunanza e la fratellanza delle varie famiglie italiane ${ }^{17}$.

21 Alla fine, la Commissione alla Camera rigetterà gli articoli 8 e 9 dell'allegato c dei Provvedimenti per il pareggio del Bilancio. Ma l'anno successivo Mongeri rilancerà lo spirito complessivo della riforma dalle pagine di Nuova Antologia. ${ }^{18}$

L'articolo dell'ex segretario dell'accademia braidense risponde puntualmente alle obiezioni presentate da quei "propugnacoli", che sarebbero, a suo avviso, le accademie di belle arti. Mongeri affronta prima di tutto il "pregiudizio" che vincola le pinacoteche all'istruzione artistica, screditando in particolar modo il valore formativo della copia, che considera pratica fuorviante. Secondo Mongeri, infatti, gli studenti imparerebbero molto di più dai quadri antichi "meditandovi, che copiando per imitare", poiché nelle pinacoteche "non si apprende che la scienza dell'arte". La dimensione scientifica delle raccolte si concretizza, infine, nell'ordinamento delle opere.

Anche su questo aspetto Mongeri contraddice la posizione degli accademici e attacca i criteri estetici, ormai desueti, delle sale dei capolavori. Per Mongeri "mettere in evidenza i contrasti, anziché le analogie", al fine di "chiarire le varietà e le differenze che dividono gli artisti" e "far meglio spiccare le grandi individualità dell'arte", è una scelta poco rigorosa e controproducente. Lo studioso milanese invece sostiene, ancora una volta, un criterio analogico, metodico e razionale, concentrato sulla rappresentazione della scuola artistica locale. Tale criterio consentirebbe agli studiosi di "cogliere l'arte nel suo procedimento storico" e assolverebbe anche una funzione educativa generale, presentando a un pubblico allargato una ricostruzione evidente e comprensibile.

Quindi, Mongeri chiede retoricamente al lettore: "le scuole locali non costituiscono di già il fondo principale onde si vantano ricche [le pinacoteche]?" Alla luce di ciò, l'ordinamento promosso asseconderebbe la naturale consistenza delle raccolte. Per raggiungere l'obiettivo basterebbe semplicemente che dei conservatori, dotati di specifiche competenze, provvedessero alla revisione delle attribuzioni, alla selezione delle opere, alla verifica dei materiali conservati nei magazzini o in deposito presso le chiese. E infine che concordassero eventuali scambi con altre istituzioni.

È proprio quest'ultimo punto a paventare tra qualche "vittima inconscia di quell'amore delle cose di famiglia", la drammatica prospettiva "che si vogliano cozzare i quadri delle Pinacoteche italiane come un mazzo di carte da giuoco per ripartirle poi a gruppi secondo i segni onde s'improntano". Ma visto che le permute verrebbero concordate dalle istituzioni stesse, Mongeri ritiene implausibile l'idea, per esempio, che lo Sposalizio della Vergine di Raffaello finisca a Firenze o a Perugia, come invece temuto dagli accademici milanesi. È da notare, però, che l'attenuazione delle ipotesi più radicali da parte di Mongeri, non implica una sua adesione alla sensibilità moderna per la sedimentazione storica delle collezioni, che è concetto ancora lontano. Nella sua logica, infatti, la rivalutazione complessiva delle esperienze, anche minori, delle diverse scuole, legittimerebbe a ignorare i timori di uno snaturamento delle raccolte.

Nel frattempo la funzione delle pinacoteche, sfiorata dall'elaborazione istituzionale, diventa argomento di dibattito anche all'interno dell'ambiente accademico. Il secondo Congresso artistico, che riunisce accademici, artisti e critici, tenuto a Milano nel 1872, 
prevede infatti una sezione dedicata all'Archeologia Artistica, con il compito di discutere, fra l'altro, i criteri di ordinamento e catalogazione dei musei. Alcuni spunti di discussione promossi dal comitato esecutivo del Congresso ricalcano puntualmente le posizioni di Mongeri e di Belgiojoso (membri dello stesso comitato e della commissione per la scelta dei temi): ci si chiede infatti se nell'ordinamento delle opere "convenga meglio mettere in evidenza le analogie e quindi, occuparsi a preferenza delle scuole e delle opere locali, ovvero mostrare le varietà ed i contrasti onde vanno contraddistinte ${ }^{19}$."

Questa volta l'assemblea nazionale degli addetti ai lavori appoggia, con qualche cautela, l'ordinamento cronologico per scuole pittoriche, senza però spingersi ad avvalorare la rappresentazione della scuola pittorica locale ${ }^{20}$.

Per quanto riguarda il dibattito sull'insegnamento, invece, il Congresso riflette anche sul ruolo del patrimonio storico-artistico nella formazione dei giovani artisti. Sullo sfondo di un dibattito tra classicismo e aggiornamento veristico, l'assemblea discute la precedenza dello studio delle opere del passato, rispetto allo studio dal vero. Resta però una certa ambiguità sul significato attribuito al termine "studiare". Nelle diverse posizioni emerse, lo studio dell'antico sembrerebbe essere comunemente inteso come copia e imitazione. Ma al termine della discussione, l'assemblea approva un ordine del giorno, presentato dallo scrittore e recensore d'arte Enrico Panzacchi, in cui si prescrive non lo studio ma l'osservazione delle opere del passato ${ }^{21}$. Una distinzione terminologica sottile ma sostanziale, come fa notare uno dei firmatari della mozione, Camillo Boito ${ }^{22}$.

29 A quanto sembra, quindi, le pinacoteche, da serbatoi di modelli, si stanno affermando, anche tra gli artisti, come luoghi di meditazione sulle dinamiche storico-artistiche. Al punto che, nel 1882, sarà la Commissione permanente di belle arti, cioè un organo consultivo ministeriale composto interamente da artisti e per metà espressione diretta delle accademie di belle arti, a promuovere l'autonomia delle pinacoteche all'interno di una proposta (naufragata) per il riordinamento dell'insegnamento artistico ${ }^{23}$.

\section{L'autonomia delle pinacoteche tra riforma delle accademie e riorganizzazione ministeriale}

Nel frattempo, un processo parallelo inizia a scalfire l'assetto delle raccolte: la riforma istituzionale delle accademie di belle arti.

31 Tra il 1869 e il 1870, la Giunta di Belle Arti, organo consultivo del Ministero della Pubblica Istruzione, lavora a un progetto di statuto generale, che, in un breve giro d'anni, ispirerà le riforme delle singole accademie del Regno ${ }^{24}$. Si tratta in sintesi di un progetto che mira a modernizzare l'insegnamento artistico secondo quanto emerso dal dibattito di settore, ma che soprattutto punta a imporre un controllo diretto e accentrato sul funzionamento scolastico, corrodendo l'autorità dei ceti accademici locali. La novità più importante, infatti, è la distinzione all'interno delle accademie di belle arti tra collegi accademici e istituti: quest'ultimi, che rappresentano la componente didattica, sono posti quindi alle dirette dipendenze del Ministero della Pubblica Istruzione.

Il progetto di statuto non specifica con chiarezza la sorte delle pinacoteche. In sede di applicazione della riforma, però, tolto l'esempio della raccolta inquadrata come 
proprietà della romana Accademia di San $\mathrm{Luca}^{25}$, è generalmente privilegiata l'assegnazione agli istituti, anche se con alcune ambiguità che necessitano più volte di chiarimenti ufficiali: come nel caso della Pinacoteca di Parma, per la quale il Direttore dell'Istituto, Francesco Scaramuzza, arriverà persino a chiedere, senza esito, l'autonomia amministrativa ${ }^{26}$.

La generale destinazione delle raccolte agli istituti segna uno stacco rispetto alla tradizionale competenza accademica, ma conferma la funzione didattica delle quadrerie. In definitiva, la riforma delle accademie ha dei riflessi perlopiù indiretti o collaterali sulla configurazione delle raccolte.

L'autonomia delle pinacoteche storiche non si realizzerà pertanto né dietro le spinte dei conoscitori per l'ampliamento della funzione delle raccolte e per il riconoscimento delle competenze storico-artistiche necessarie alla loro gestione, né al termine di una riflessione istituzionale sul rapporto tra patrimonio storico-artistico e istruzione artistica. La spinta decisiva consisterà piuttosto nell'esigenza di una razionalizzazione amministrativa.

L'autonomia delle pinacoteche viene infatti intesa come precondizione necessaria per dare un ordinamento uniforme al personale coinvolto nella gestione delle raccolte statali, fino a quel momento a diverso titolo dipendenti dal Ministero della Pubblica Istruzione. Il 13 marzo 1882, insieme al decreto che sancisce la separazione delle raccolte dagli istituti di istruzione, è emanato infatti anche il primo il Ruolo unico del personale addetto a Musei, alle Gallerie, agli Scavi e ai Monumenti nazionali ${ }^{27}$. Da notare, inoltre, come il nuovo stato di cose semplifichi la gestione diretta da parte del Ministero degli introiti derivati dalla tassa d'ingresso, introdotta con regio decreto del 1875 anche nelle pinacoteche delle accademie di belle $\operatorname{arti}^{28}$.

\section{Conclusioni}

Il principio di autonomia delle quadrerie storiche sottende, come si è visto, articolate motivazioni culturali; tuttavia l'effettiva separazione dalle accademie si concretizza soprattutto per esigenze di carattere amministrativo. Inizialmente, poi, il cambiamento è solo nominale. Le raccolte restano a tutti gli effetti all'interno dell'influenza accademica, mantengono le proprie sedi tradizionali (il trasferimento della Pinacoteca di Modena, già fisicamente separata dall'istituto accademico, nella nuova sede del Palazzo dei Musei avverrà nel 1894) e il ruolo di Direttore è assegnato a professori o ex cariche accademiche ${ }^{29}$. Ma sono i Regi Decreti del 1882 a consentire la successiva affermazione alla direzione delle Gallerie di personalità come Corrado Ricci e Giulio Cantalamessa, studiosi a cui si deve l'effettivo aggiornamento scientifico delle pinacoteche. Una minuta ministeriale del 1896, riconducibile al pugno di Adolfo Venturi, il fondatore della storia dell'arte come disciplina universitaria in Italia ${ }^{30}$, elogia i risvolti positivi della riforma, sia sul piano puramente amministrativo, sia su quello scientifico:

\footnotetext{
Amministrativamente, quel Decreto Reale recò una utilità grandissima perché determinò l'esistenza delle gallerie e dei musei medievali e moderni i quali non avevano i cataloghi del loro patrimonio, non generali e costanti norme per il loro funzionare. Scientificamente, quelle istituzioni presero vita, si accrebbero secondo un disegno razionale, si ordinarono secondo icriteri che la scienza va determinando mano a mano ${ }^{31}$.
} 

accademica e mondo della tutela si è prestato, commento della riforma del Ministro Pasquale Villari, che nel 1891 scindeva le division ministeriali competenti su Arte Contemporanea e Arte Antica, lo studioso Andrea Emiliani ha osservato come, "nel momento in cui le accademie di belle arti verranno distaccate dalla tradizionale convivenza con gli uffici della conservazione e della museografia (1891), il mondo dell'unità delle arti sembrerà finito per sempre ${ }^{32}$ ". Si perde così, a suo avviso, il "contatto con il peso specifico e con la materialità delle cose d'arte. $\mathrm{E}$ con questo, anche ciò che avvicinava l'atto della creazione al suo risarcimento critico, che univa l'invenzione moderna alla meditazione storica".

\section{ALLEGATO}

\section{Abbreviazioni fonti d'archivio}

AABAF = Archivio dell'Accademia di Belle Arti di Firenze.

ACS, MPI, AA. BB. AA. = Archivio Centrale dello Stato, Ministero della Pubblica Istruzione, Direzione Generale Antichità e Belle Arti.

ACS, MPI, CSPI = Archivio Centrale dello Stato, Ministero della Pubblica Istruzione, Consiglio Superiore della Pubblica Istruzione.

\section{NOTE}

1. Decreto del Commissario Straordinario Carlo Boncompagni, 21 giugno 1859, Atti e Documenti editi e inediti del Governo della Toscana, Parte prima, Firenze, 1860, p. 244-245.

2. Legge 24 giugno 1860, n. 4135.

3. Decreto del Dittatore delle Due Sicilie Giuseppe Garibaldi, 12 settembre 1860, Atti del Governo estratti dal Giornale Ufficiale di Napoli, n. 3, dal 13 al 17 settembre 1860, p. 42. 
4. Decreto del Dittatore Farini, 22 aprile 1859; BolLATI Emmanuele, Fasti legislativi e parlamentari delle rivoluzioni italiane nel secolo XIX. vol. II, 1859-1860. Parte I, Lombardia, Emilia, Milano, 1865, p. 583. 5. La raccolta, esposta nel Palazzo Ducale, è composta da materiali provenienti dalle Regie Gallerie fiorentine, concesse per volere del Granduca Leopoldo intorno al 1850 e affidate al Presidente della Commissione d'incoraggiamento e dell'Istituto di Belle Arti. La raccolta resterà dipendente dall'Istituto di Belle Arti fino al 1875, quando diventerà di proprietà comunale.

6. Nel 1863 le raccolte dell'Università di Palermo passeranno sotto l'autorità della Commissione di antichità e belle arti. Le raccolte rimarranno nel Palazzo dell'Università fino a quando, a seguito degli incrementi derivati dalle soppressioni ecclesiastiche del 1866, il Regio Museo sarà destinato ad una sede più spaziosa nella Casa dei Padri Filippini all'olivella e nel 1873 l'istituto acquisirà il titolo di Museo Nazionale.

7. "Le Gallerie, le Pinacoteche e i Musei archeologici, annessi alle Università, alle Accademie e Istituti di belle arti, cesseranno di far parte dei detti Istituti scientifici od artistici, ed avranno un'amministrazione propria, con impiegati compresi nel ruolo unico del personale stabilito con altro Nostro Decreto di pari data", regio decreto 13 marzo 1882, n. 678, che separa dagli Istituti d'insegnamento le Gallerie, le Pinacoteche ed i Musei.

8. POMIAN Krzysztof, «Le museé face à l'histoire ", in S. N., L'Histoire au musée, atti del convegno (Versailles, château de Versailles, 10-12 décembre 1998), Arles, Actes Sud, 2004, p. 99-126.

9. Il discorso è pubblicato in appendice a Marisa DALAI EMILIANI, « Giovanni Morelli e la questione del Catalogo Nazionale: un episodio poco noto della politica di tutela nell'Italia dell'Unità », in Giacomo AGostI, Maria Elisabetta MANCA, Matteo PANZERI (dir), Giovanni Morelli e la cultura dei conoscitori, atti del convegno (Bergamo, 1987), Bergamo, 1993, p. 123-128.

10. Giovanni Battista CAVAlCASELLE, «Sulla conservazione dei monumenti e degli oggetti di belle arti e sulla riforma dell'insegnamento accademico. G. B. Cavalcaselle al signor Ministro della pubblica istruzione » (1863), Accademia Clementina. Atti e Memorie, $\mathrm{n}^{\circ}$ 20-21, 1987, p. 85-112. Per un'analisi del documento si rimanda a: Donata LEVI, Cavalcaselle. Il pioniere della conservazione dell'arte italiana, Torino, 1988, p. 309-322; Andrea EMILIANI, "Giovanni Battista Cavalcaselle politico. La conoscenza, la tutela e la politica dell'arte negli anni dell'unificazione italiana », in Anna Chiara TOMMASI (dir), Giovanni Battista Cavalcaselle conoscitore e conservatore, Venezia, 1998, p. 323-369.

11. Cavalcaselle al Ministro della Pubblica Istruzione, Carlo Matteucci, 24 agosto 1862, in Donata LEVI, «Storiografia artistica e politica di tutela: due memorie di G.B. Cavalcaselle sulla conservazione dei monumenti (1862) ", in Simonetta LA BARBERA (dir), Gioacchino di Marzo e la critica d'arte nell'Ottocento in Italia, atti del convegno, (Palermo, 2003), Bagheria, 2004, p. 74.

12. Giovanni Morelli a Gaetano Giordani, 28 luglio 1862, in Gian Piero CAMMAROTA, Le origini della Pinacoteca Nazionale di Bologna. Una raccolta di fonti, vol. II, Dalla rifondazione all'autonomia (1815-1907), Bologna, 2004, p. 417.

13. Giuseppe MONGERI, «Le belle arti davanti al Parlamento italiano », La Perseveranza, $n^{\circ} 966,24$ luglio 1862 , p. 3.

14. Proposte d'ordinamento per le Pinacoteche e i Musei Nazionali, 9 febbraio 1870, in ACS, MPI, AA. BB. AA., I versamento, Musei, Gallerie e Pinacoteche, b. 170, fasc. 1-1.

15. ATTI PARLAMENTARI, Camera dei Deputati, X Legislatura, sessione 1869-1870, Documenti, n. 53, Provvedimenti pel pareggio del bilancio presentati dal Ministro delle Finanze (Sella) nella tornata del 10 marzo 1870, Allegato C, Legge sull'istruzione pubblica, art. 9, p. 172.

16. Il Presidente dell'Accademia di Belle Arti di Milano, Carlo Belgiojoso, al Presidente dell'Accademia di Belle Arti di Firenze, Niccolò Antinori, 12 aprile 1870, in AABAF, filza 1870.

17. Delibera del Consiglio accademico di Venezia, 18 aprile 1870, in ACS, MPI, AA. BB. AA., Istituti di belle arti, b. 6 . 
18. MONGERI Giuseppe, «Dell'ordinamento delle pubbliche pinacoteche in Italia », Nuova Antologia, vol. $17, \mathrm{n}^{\circ} 5,1871$, p. 57-78.

19. Atti del secondo Congresso Artistico italiano (Milano, 1872), Milano, 1874, p. 15.

20. "Si toccò pure del miglior modo di ordinare le pinacoteche: e pur riconoscendo ch'esse generalmente devono servire alla natura dei locali, alla grandezza dei dipinti e alle disposizione precedenti, si trovò desiderabile la distribuzione per scuole, e queste per età." Atti del secondo Congresso Artistico italiano, 1874, p. 190.

21. "I sottoscritti opinano che quando un giovane sia giunto al grado di poter ritrarre dal rilievo le forme degli oggetti in genere, con sufficiente esattezza e con giusta intelligenza delle ragioni della prospettiva e del chiaroscuro, [...] debba passare anzi tutto allo studio del vero; intendendo per vero i gessi ricavati dal naturale, i panneggiamenti, il modello dal vivo, il paese ecc. Ma riconoscendo in pari tempo essere sommamente opportuno che gli esempi dei buoni artisti stiano sempre esposti alla vista degli studiosi per utili confronti, propongono il seguente ordine del giorno: Che i giovani debbano fare camminare di pari passo lo studio e l'imitazione del vero, colla osservazione delle opere dei buoni artisti." Atti del secondo Congresso Artistico italiano, 1874, p. 103.

22. "Il nostro ordine del giorno dice 'osservare' le opere dei grandi maestri e non 'studiare', quello del signor Villa dice 'studiare' e non 'osservare'. La differenza qui è essenzialissima e importa farla notare, perché non si dica che abbiamo voluto far passare il nostro ordine del giorno con degli equivoci". Atti del secondo Congresso Artistico italiano, 1874, p. 104.

23. Per i lavori della Commissione si veda ACS, MPI, AA. BB. AA., Istituti di belle arti, b. 3.

24. Due versioni del progetto si conservano in ACS, MPI, CSPI, Giunta di Belle Arti, b. 59, fasc. 59. Per l'inquadramento dei lavori della Giunta si veda Marica MARzINOTTo, «Giovanni Battista Cavalcaselle 'nemico' della Pontificia Accademia di San Luca ", Ricerche di storia dell'arte, $\mathrm{n}^{\circ} 107$, 2012 , p. 47-58. I punti principali del progetto sono recepiti in via sperimentale nello statuto dell'Accademia di San Luca a Roma e in quello dell'Accademia delle Arti e del Disegno di Firenze, emanati il 9 ottobre 1873 e il 3 novembre 1873 (modificati il 22 e 29 giugno 1874 e ancora il 3 dicembre 1876). La riforma sarà poi estesa alle accademie di belle arti di Modena, Bologna e Parma, con regio decreto del 23 settembre 1877, e all'Accademia di Belle Arti di Venezia, con regio decreto dell' 8 settembre 1878. Sulle riforme si veda: Camillo вогто, «I nuovi decreti sulle accademie di belle arti », Nuova Antologia, vol. XXV, n 4, aprile 1874, p. 880-896; Luciano CARAMEL, « Le accademie artistiche italiane nel corso del XIX secolo: una lunga crisi di identità », Ottocento. Catalogo dell'arte italiana dell'Ottocento, $\mathrm{n}^{\circ}$ 18, 1989, p.27-30; Concetto NICOSIA, Arte e Accademia nell'Ottocento. Evoluzione e crisi della didattica artistica, Bologna, 2000, p. 204-209; Pietro ROCCASECCA, " Appunti per una storia della formazione accademica degli artisti (1754-1923) ", in Costanza BARBIERI, The lost art of drawing. Disegni inediti di Architettura dal Fondo Storico dell'Accademia di Belle Arti di Roma, cat. esp. (Roma, Centro Studi Americani, 2016), Roma, 2016, p. 61-66.

25. L'Accademia di San Luca, privata delle funzioni didattiche con regio decreto 29 giugno 1874, risulta autonoma dal Ministero delle Pubblica Istruzione. Le collezioni, profondamente intrecciate con la storia dell'istituzione, non saranno pertanto toccate dal decreto del 1882 .

26. Si veda la corrispondenza in ACS, MPI, AA. BB. AA., I versamento, Musei, Gallerie e Pinacoteche, b. 306, fasc. 169-3. Nel caso veneziano, invece, la destinazione delle raccolte all'Istituto è in parte revocata con regio decreto del 9 novembre 1879.

27. Regio decreto 13 marzo 1882, n. 679, (serie 3), che approva il ruolo unico degli impiegati addetti ai Musei, alle Gallerie, agli Scavi ed ai Monumenti Nazionali. I decreti del 13 marzo seguono un ripensamento della ripartizione di competenze a livello ministeriale. Su questo versante, va ricordata innanzitutto la creazione nel 1875, della Direzione Centrale degli Scavi e dei Musei del Regno. Il nuovo ufficio tecnico è affidato all'archeologo Giuseppe Fiorelli, che sarà il regista di un sistematico intervento di razionalizzazione nella gestione dei musei di antichità. Tra gli interventi più significativi si segnala la stesura nel 1878 del Ruolo organico e Regolamento pel servizio 
dei musei di antichità dello Stato, in Daniele JALLA, Il museo contemporaneo. Introduzione al nuovo sistema museale italiano, Torino, 2003, pp. 65-69. Con la creazione, nel 1881, della Direzione Generale per le Antichità e Belle Arti, anche il settore artistico viene assegnato alla direzione di Fiorelli che può così estendere la sua politica accentratrice anche ai musei artistici.

28. Si veda: Martina LERDA, La politica museale dopo l'Unità: l'introduzione dell'ingresso a pagamento nei musei statali (1860-1875), tesi di laurea, Università di Pisa, Antonella GIOLI, Ettore SPALLETTI (dir), 2010; Antonello RICCO, "Tasse e tessere d'ingresso in musei, gallerie, scavi e monumenti governativi del Regno d'Italia (1875-1939) », Aedon, n 3, 2011.

29. Il ruolo di Direttore è ricoperto a Bologna da Cesare Masini, a Milano da Giuseppe Bertini, a Modena da Adeodato Malatesta, a Venezia da Nicolò Borazzi e a Parma da Cecrope Barilli, contemporaneamente Direttore dell'Istituto di Belle Arti. Diverso il caso fiorentino dove la competenza sulle raccolte ex-accademiche è trasferita alla Soprintendenza delle Regie Gallerie.

30. Si veda: Giacomo AGosti, La nascita della storia dell'arte in Italia. Adolfo Venturi dal museo all'università 1880-1940, Venezia, 1996; Mario D'ONOFRIO (dir), Adolfo Venturi e la storia dell'arte oggi, Modena, 2008.

31. Ministero della Pubblica Istruzione al Segretario di Stato per la Pubblica Istruzione, 7 aprile 1896, in ACS, MPI, AA. BB. AA., II versamento, III parte, b. 118.

32. EMILIANI, « Giovanni Battista Cavalcaselle politico... », 1998, p. 327-328.

\section{RIASSUNTI}

Nel 1882 un regio decreto del Regno d'Italia sottrae alle accademie di Belle Arti la gestione delle pinacoteche storiche, che diventano istituti autonomi, direttamente dipendenti dal Ministero della Pubblica Istruzione. Sebbene di carattere prevalentemente amministrativo, questa importante cesura sancisce ufficialmente un cambiamento nel rapporto tra arte del passato e istruzione artistica, che si verifica in significativa concomitanza con il processo di affermazione della Storia dell'Arte come disciplina autonoma. Da strumenti didattici e repertorio di modelli per i giovani pittori, le pinacoteche si vanno configurando a livello istituzionale come luoghi di studio, di osservazione e di meditazione sugli sviluppi storico-artistici.

Il saggio ripercorre le complesse dinamiche di questo processo, in cui agiscono spinte differenti: dalle rivendicazioni disciplinari dei primi conoscitori, agli interventi di riforma dell'insegnamento artistico, sino alle esigenze di riorganizzazione amministrativa del sistema museale statale.

\section{AUTORE}

\section{MARTINA LERDA}

martina.lerda@alice.it 